\title{
Overview Of A Large Scale Monitoring Project Of Energy Positive Houses: Complementarity Between Simulations And Measurements
}

\author{
Jeanne Goffart ${ }^{1}$, Monika Woloszyn ${ }^{1}$, Xavier Faure ${ }^{2}$, Frederic Wurtz ${ }^{3}$, Léa Gondian ${ }^{1,7}$, Catherine \\ Buhé $^{1}$, Thomas Recht ${ }^{4}$, Laurent Mora ${ }^{4}$, Bruno Peuportier ${ }^{5}$, Patrick Schalbart ${ }^{5}$, Manar Aymari ${ }^{6}$, \\ Stéphane Ploix ${ }^{6}$, Patrice Schneuwly ${ }^{7}$, Etienne Wurtz ${ }^{7}$ \\ ${ }^{1}$ LOCIE, CNRS, Univ. Savoie Mont Blanc, France \\ ${ }^{2} \mathrm{CEA}$ Tech, France \\ ${ }^{3}$ Univ. Grenoble Alpes, CNRS, Grenoble INP, G2Elab, 38000 Grenoble, France \\ ${ }^{4}$ I2M, CNRS, Univ. Bordeaux, France \\ ${ }^{5}$ MINES ParisTech, PSL Research University, CES, France \\ ${ }^{6} \mathrm{G}-\mathrm{SCOP}$, CNRS, INP, Univ. Grenoble Alpes, France \\ ${ }^{7}$ CEA, LITEN, INES, Univ. Grenoble Alpes, France
}

\begin{abstract}
The present paper gives an overview of a French national project on building performance. Named COMEPOS, started in 2013, it focuses on « Positive Energy » detached houses.

It consists in the design, construction, operation and evaluation of 20 inhabited and innovative houses. This collaborative project includes constructors, industrial and academic partners, the latter involved both in the monitoring and the use of building performance simulation at different stages of the building's life. The paper offers an overview of how simulation technics can be used, in coordination with measurements, concerning points like the optimization of the design, performance evaluation, fault detection, but also improving simulation of real-life situations, for example by improving the knowledge about the impact of occupants' behaviour.
\end{abstract}

\section{Introduction: context and objectives of the COMEPOS project - Toward Energy Positive Buildings design, construction and evaluation}

In France, building is the most energy-consuming sector. To reach the objectives of reduction of energy consumptions that France set, sector of the individual house, which represents approximately $60 \%$ of annual housing constructions, establishes an essential stake. Political commitments leading the building sector to generalize "positive energy houses" (that is to say, producing more energy than they consume over a year) in 2020 are ambitious. Research efforts and demonstration remain necessary to define and validate the concept of energy-plus houses, in terms of characteristics and expected requirements.

The COMEPOS project involves 22 partners. It consists in developing the concept of detached energy-plus houses for all energy uses (regulatory uses and uses said "movable" not regulatory) with a real control of the additional costs, within the framework of the evolution of the thermal regulations on the horizon 2020. It has two main objectives:

- Realize design studies, simulation, technological design, monitoring and experience feedback of demonstrators, as well as the development of innovative technological systems that may be integrated;

- Demonstrate the feasibility of the concept through the construction of 20 demonstration buildings that are distributed throughout the French territory, in partnership with a dozen builders who are members of LCA-FFB (Builders of individual homes Union).

The large and dispersed sample over the entire territory of these demonstrators, integrating the various innovations proposed by industrials, can help better define effective solutions depending on climate types, building patterns but also lifestyles and clients' expectations.

The project allows mutualizing between the various partners the know-how and the following innovations:

- Analysis of the performances of already built energyplus buildings, evaluation and choice of technological components best-suited for each region;

- Houses design (building energy performance simulation, Life cycle analysis tools), taking into account various builders practices and expectations;

- Houses construction: definition of an economic, commercial and legal model and elaboration of a guarantee of energy performance;

- Monitoring in exploitation phase (analysis of measurements and inquiries with construction actors and occupants).

The project will help accelerate the market development for energy-plus houses, HVAC systems and innovative components, as well as optimized design and energy management. As a result of first demonstrators during the project, the large-scale development of this type of home is expected, first of all by the different builders 
partners in the project, and then in the longer term, by all members of LCA-FFB.

This paper especially focuses on scientific developments made during the project. Those developments cover a great part of the life-cycle of energy-plus building and that are respectively:

- Ecodesign;

- Deployment of monitoring systems and on-site measurement;

- Global data analyses;

- Calculation of global indicators from measurements;

- Comparison of predicted simulated values versus measured performances;

- Measurement for default diagnostic and guaranty of performances.

\section{Ecodesign of 'energy-plus houses' using multi-objective optimisation, life cycle assessment and stochastic occupancy model}

Designing energy-plus buildings, at lower environmental impact and lower cost, is a complex optimisation problem. To address this issue, an ecodesign approach associating multicriteria optimisation, stochastic occupancy model, and simulation tools (for energy and environmental performance) was developed and applied in the COMEPOS project.

\section{Methodology}

A challenge in the design phase is to explore a large range of solutions in a limited time, in order to optimize the performance according to several criteria that can be contradicting. The optimisation procedure can be computationally expensive, especially when the number of dimensions of the search space is high. In that case, selecting suitable design variables constitutes an interesting preparatory stage of optimisation (Machairas et al., 2014). This selection stage can be carried out using sensitivity analysis, e.g. Morris screening method (Morris, 1991). Expert judgment is a complementary way to simplify the optimisation problem by identifying the most influential design variables.

The optimization stage is conducted using a genetic algorithm, NSGA-II (Deb et al., 2002). As a simplified computing transposition of Darwin's theory, the goal is to mimic a living organism's population adapting itself to its environment over generations. Starting with a random initial population of $\mu$ individuals, $\lambda$ individuals are selected by tournament (step 1 in Figure 1). These individuals, called "parents", generate $\lambda$ "children" via stochastic operators representing the crossover and mutation phenomena in genetics (step 2). The children's performance is evaluated (step 3) in order to create a new generation of $\mu$ individuals using an elitist selection between the current $\mu$ individuals and the $\lambda$ children (step 4). The process is generally stopped when reaching a given number of generations, but can also be defined regarding the current population's performance.

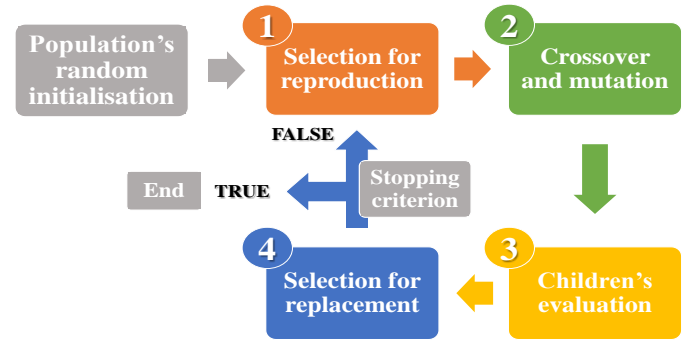

Figure 1: Illustration of the genetic algorithm process

During the design process of plus-energy houses, the target of the COMEPOS methodology was to find a prototype with a positive annual primary energy balance which jointly minimises construction cost and greenhouse gases emissions (in $\mathrm{CO}_{2}$ equivalent). To assess the energy performance, the dynamic building energy simulation software COMFIE, created by Peuportier and Blanc-Sommereux (1990) was used. The model's reliability was studied in the particular context of a high energy performance house (Brun et al., 2009; Munaretto, 2013; Recht et al., 2014 ; Munaretto et al., 2018). The building environmental performance was assessed using an LCA software (EQUER), created by Polster (1995) and coupled to COMFIE. The construction, exploitation, renovation, and demolition stages were taken into account. Life cycle simulation (annual time step) uses the ecoinvent database to establish an inventory of flows from and into the environment. Construction cost functions were developed in collaboration with constructor partners.

Being more and more insulated, high energy performance buildings are significantly sensitive to internal loads (metabolism, electrical appliances), commonly modelled by conventional ratios and profiles. In the COMEPOS project, average statistical occupancy profiles based on a stochastic occupancy model (Vorger et al., 2014) were generated, allowing a more realistic consideration of occupants' behaviour.

\section{Illustration}

For illustrative purposes, the results obtained during the design phase of one COMEPOS house are presented in Figure 2. Eleven design variables were considered: insulation thicknesses of the walls (1), the roof (2), and the floor (3), areas of four south-east or south-westfacing windows $(4,5,6,7)$, the type of glazing of northeast-facing windows (8), the type of the mechanical ventilation system (9), the installation (or not) of a greywater heat recovery system (10), and the number of photovoltaic modules (11). These variables, divided into levels between 2 and 16 (depending on the variable), lead to a search space of several millions of solutions. In approximately two hours, the algorithm identified a solutions set near the theoretical one. The analysis of characteristics provides useful information regarding main questions in design phase that are "which repartition of investment between envelope and equipment?" and "when several criteria are addressed, are there some prevailing solutions or only compromises?". 


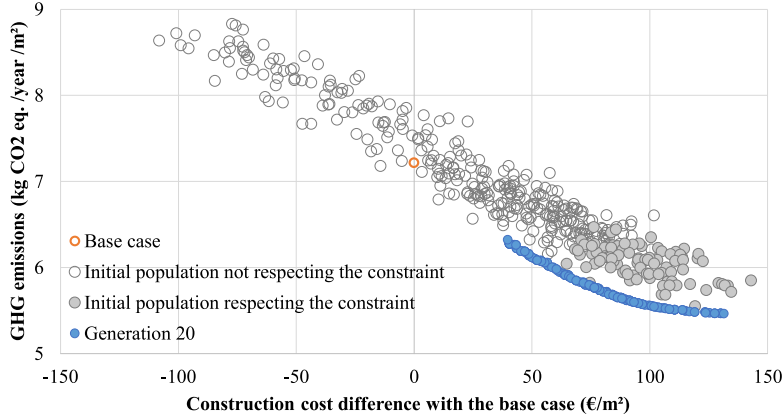

Figure 2: Base case, initial population and final Pareto front

\section{Conclusion and perspectives of the proposed design} approach

An ecodesign approach associating multicriteria optimization, stochastic occupancy model, and simulation tools was developed, contributing to the decision making process, beyond simple evaluation by simulation. Solution robustness regarding occupancy diversity and climate change was also studied within the project (Recht et al., 2016).

\section{Monitoring systems deployed in constructed demonstrators}

One main goal of the project is the development of systems to manage all house equipment (HVAC, bindings, natural ventilation openings, etc.) and to produce energy consumption dashboards adapted to all types of operation. Two levels of systems have been developed and studied:

A standard level, looking for the best compromise between cost and features, and a more complex level allowing to minimize building energy consumption through innovative control algorithms.

The project will allow the pooling of the knowledge of builders of individual houses on the BEPOS and demonstrators' realization on the scale of the territory.

\section{Environment}

All the different experimental sites are equipped with a common base of sensors. Some additional sensors have been added in specific demonstrators depending on the installed innovative systems characteristics.

Two industrial partners were involved in the sensor networks and communication for data collection, what results in two different architectures presented in Figure 3. Both are able to aggregate several communication protocols and, thus, the use of almost all kind of sensors. For non-intrusive reasons and following the project objectives, wireless industrial sensors were installed as much as possible. When available, the equipment external communication protocol was used (like typically MODBUS). This was necessary for equipment that produces both heating and domestic hot water for example in order to correctly identify the electric consumptions for each service.

The common base data collection consisted in measuring electric consumption for heating, lighting, domestic hot water production and domestic activities. Ambiance parameters (temperature and relative humidity) were measured in all living and service rooms. $\mathrm{CO}_{2}$ concentration was measured in at least two main zones (main living room and main bedrooms). And in some sites, full volatile organic compounds (VOCs) sensors were also added.

Complement monitoring systems were needed for specific sites that integrate either innovative ventilation systems that required measurement of $\mathrm{CO}_{2}$ concentration in each room, innovative heating/cooling generation and distribution as a couple hydraulic ceiling and air energy distribution or solar thermal panels with high volume storage and direct distribution (using the dwelling mass as storage device) which both require calorific energy measurements. Sites equipped with photovoltaic panels and, for some, coupled with an electrochemical storage device, also required specific electric power sensors.

One of the challenging aspects for the sensor network was to integrate its installation phase within the global building process for each site. Some sensors were installed within the plumbing network while some others were installed in the electric main board, and, finally, the ones related with internal ambiance, were required to be installed after occupants arrived in the dwelling. Thus full sensor network was installed in quite long time range and by many different stakeholders, leading for some site to quite difficulties in order to get it fully functional.

For all sensors, data collection is ensured every minute. Analyses are then performed with oversampling, if needed, with one-minute time step, leading to 1440 values each day for each sensor.

Data are not mean values over the minutes but instantaneous values.

Depending on the manufacturer's architecture, the data are stored every day on main server for analyses.

\section{Some global data analyses resulting from measurements}

Building energy and indoor ambiance simulations need accurate inputs, especially in the context of energy performance contracting (Faggianelli et al, 2017). These are needed for all simulations from the simplest model with one single inside node and mean envelope effect to multi-zone models with highly detailed envelop description. The panel of uncertainties origin spreads from building characteristics (thermal bridges, air tightness and its localisation ...) to occupant behaviour (zone target temperatures, presence, activities ...). The different sites and long-time acquisitions (two years) enable to get global views on occupant behaviour and presence.

As an example, $\mathrm{CO}_{2}$ concentration in main rooms enables to get an estimate of indoor air quality with respect to bio-effluents and gives some valuable information on occupancy. These are useful indications for simulation purposes. Figure 4 presents heatmaps of $\mathrm{CO}_{2}$ concentration in the main room of several sites. The 

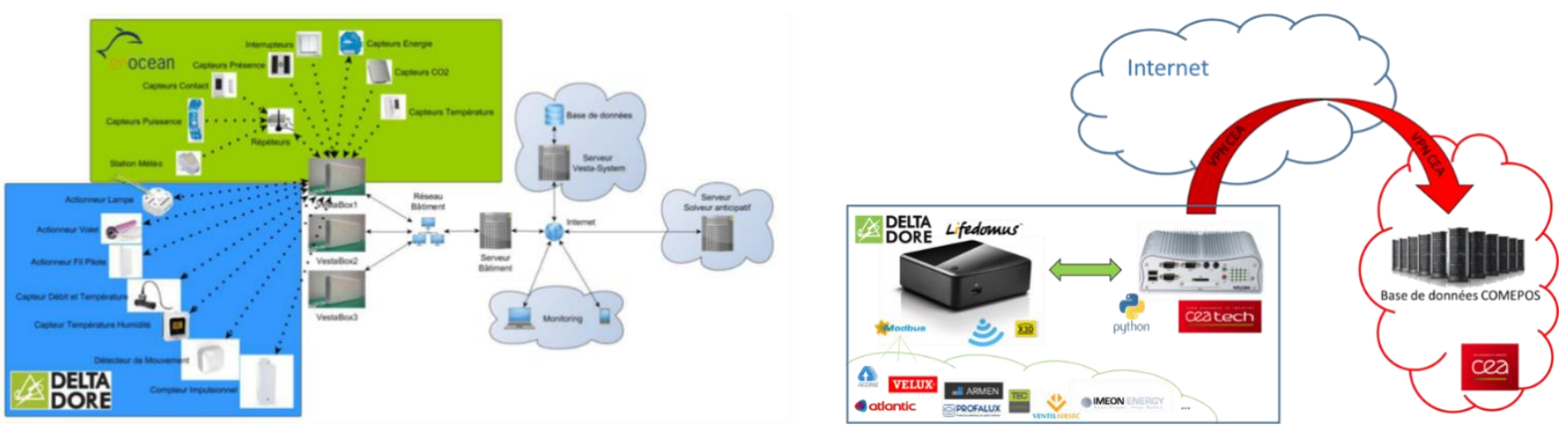

Figure 3: 2 sensor network architecture and data collection (right scheme)

\section{CO2 concentration in main bedroom of 6 different occupied dwellings}
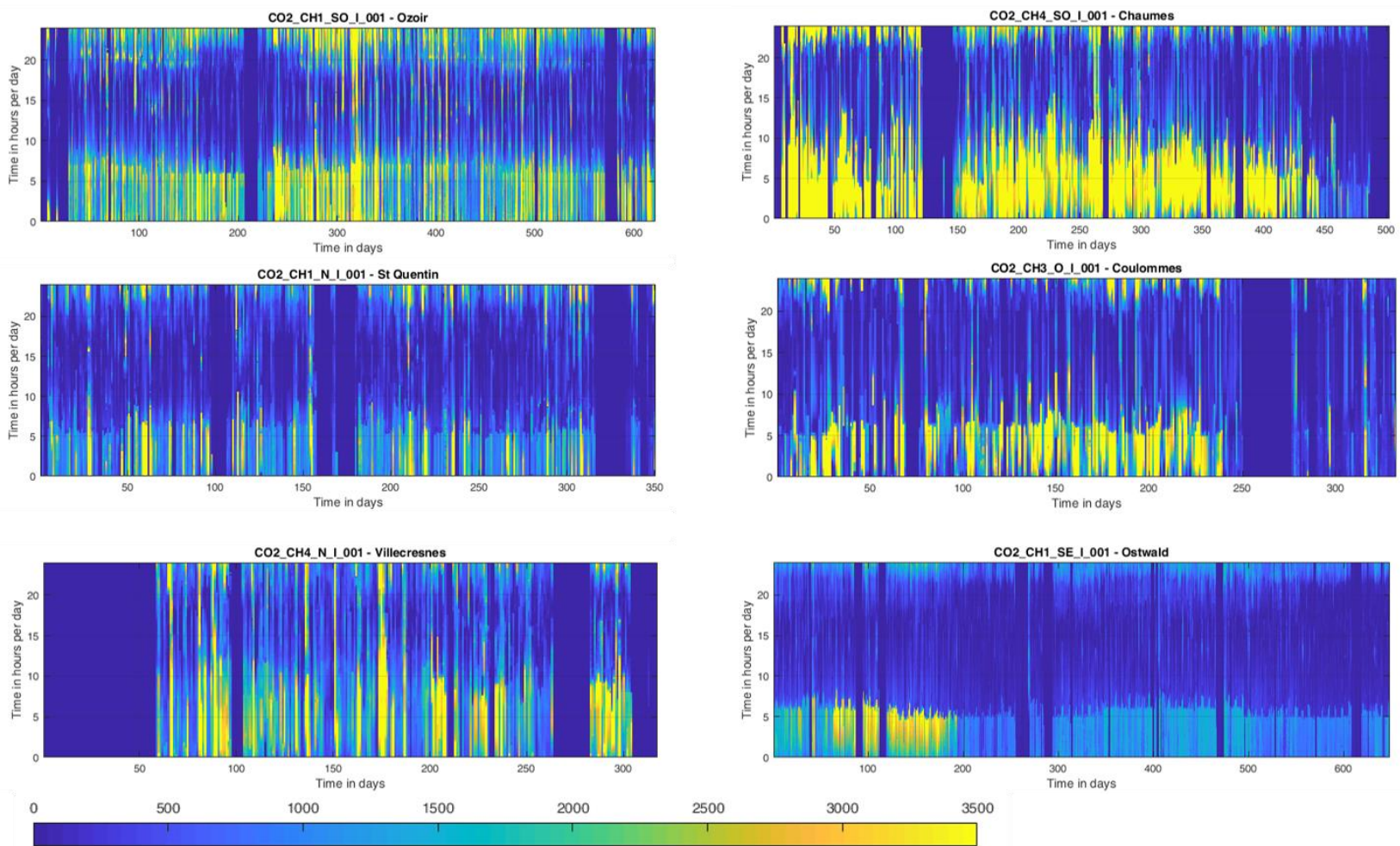

Figure 4: Heat maps of CO2 concentration in main rooms of several sites

vertical axis starts at 12:00 a.m. and ends at 11:59 p.m. every day along the horizontal axis. This representation enables to easily catch the repeatability of occupant behaviour (presence and wake-up). In one specific heatmap, one can see the winter/summer hour change as well as the difference between weekends and working days.

Collected data for domestic activities and lighting might be treated the same way and computed in a compatible statistical form for numerical simulation enhancement.

Indeed, in order to get a step forward guaranteed performances, a stochastic parts of inputs is needed to compute an effective performance range since even if occupant have quite same attitude, the inherent variability automatically leads to performance's variability.

\section{From measurements to indicator's calculation based on INDICOM as common framework}

INDICOM is a software tool developed in Python as an open source application to ease the measurement data analysis and to compute different indicators for the positive energy houses of the COMEPOS project.

Since the monitored houses are equipped with sensors to measure variables such as temperature, illumination, PV production, total consumption, etc. The aim of INDICOM is to connect permanently the data coming from the sensors.

The data collected from the project's houses need to be analyzed and filtered. Thus, noises and outliers are removed from the dataset: it's one feature of INDICOM.

Besides, INDICOM has been built to evaluate the energy performance of the connected houses as well as to 
monitor the comfort levels by calculating different types of indicators, and proposing by this way a common referential in order to compare the performances of each houses, and this for energy and comfort performances.

Indicators can also be communication means to interact with occupants of a living area so they can take informed decisions regarding their everyday practices and customs. Consequently, occupants are able to compare and challenge with others or with their past results. Moreover, occupants will be able to understand the consequences and effects of their behavior regarding energy and learn how to improve it.

INDICOM is organized into three main folders: a folder for indicators independently of any sites, a folder for sites with connections with data and relevant processings and finally, a folder for coupling indicators with each site.

As an example, the first demonstrator of the COMEPOS project was equipped with approximately 200 sensors, which provide data on air quality, energy consumption and PV panel.

On this demonstrator, the following match-load indicators have been calculated for different time windows (or time scale) (day, week, month and year):

- Self-consumption: it corresponds to the share of photovoltaic electricity production that is consumed on a site instantly. It has a value between 0 and 1.0 means nothing of the site generation is used by the load (no load), while 1 means that all the energy produced on the site is consumed on the site. The values of this indicator are the lowest during summer and highest during winter. This is due to the low PV production and higher electricity consumption in winter (i.e., for 4 months in summer total consumption is equal to $547 \mathrm{kWh}$, and total production is equal to $834 \mathrm{kWh}$, while for the same house for 4 months in winter, total consumption is equal to $827 \mathrm{kWh}$, and total production is equal to $175 \mathrm{kWh})$

SelfCons $=\frac{\int_{t_{1}}^{t_{2}} \min [g(t)-s(t)-\zeta(t), l(t)] d t}{\int_{t_{1}[g(t)-s(t)-\zeta(t)] d t}^{t_{2}}}$

Where $g(\mathrm{t})$ is the onsite production, $s(\mathrm{t})$ is the storage energy balance, $\zeta(\mathrm{t})$ energy losses within the system $l(\mathrm{t})$ building load (demand).

- Self-production: it has a value between 0 to 1 , where a value of 1 means that all the load is supplied by the on-site production and 0 means that none of the load is supplied by the on-site production.

This value increases in summer and drops in winter cause to lower PV production and higher consumption.

$$
\text { SelfProd }=\frac{\int_{t_{1}}^{t_{2}} \min [g(t)-s(t)-\zeta(t), l(t)] d t}{\int_{t_{1}[l(t)] d t}^{t_{2}}}
$$

- Coverage rate: it provides information about the energy balance of the house over a given time period values more than one means a positive energy house.

$$
\text { Coverage rate }=\frac{\int_{t_{1}}^{t_{2}} g(t)}{\int_{t 1^{[l(t)] d t}}^{t_{2}}}
$$

- Loss of load probability: It gives an indication of the dependence of the building on the grid and has a value between 0 to 1 : 1 will indicate a full dependence on the grid for the time of study and 0 will indicate a full autonomy from the grid for the period of study (Salom, 2014).

$$
L O L P=\frac{\int_{t_{1}}^{t_{2}} T d t_{l(t)>(g(t)-s(t)-\zeta(t))}}{t_{1}-t_{2}}
$$

In addition, different usage indicators have been calculated in order to analyze occupant behavior towards energy usage. Heat flow through door/window indicators (heat wastes), and dish-washer/clothe-washer indicators have been integrated into INDICOM.

Moreover, in order to fully appreciate the results, INDICOM has been developed to plot all the sensors data and calculated indicators in three simple steps (see Figure 5):

1. Select the house;

2. Choose the sensor data or indicators;

3. Plot the curves.

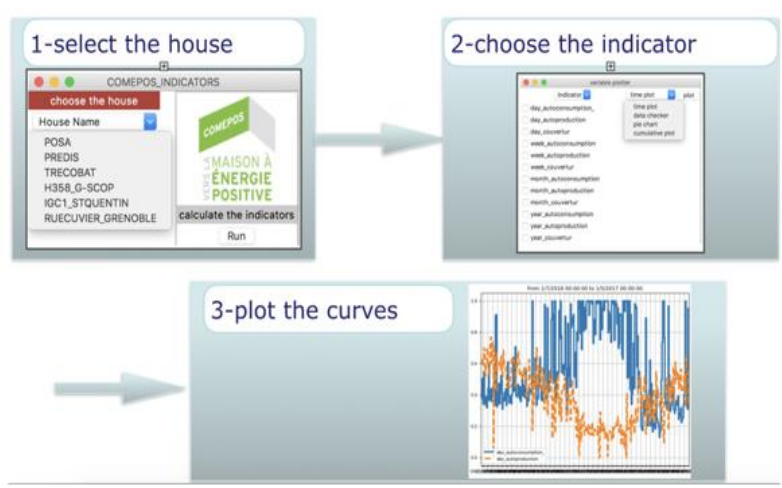

Figure 5: INDICOM interface

Figure 6 shows the results of coverage rate indicator for one year from July 2016 to July 2017 in different time scales (day, week, month and year).

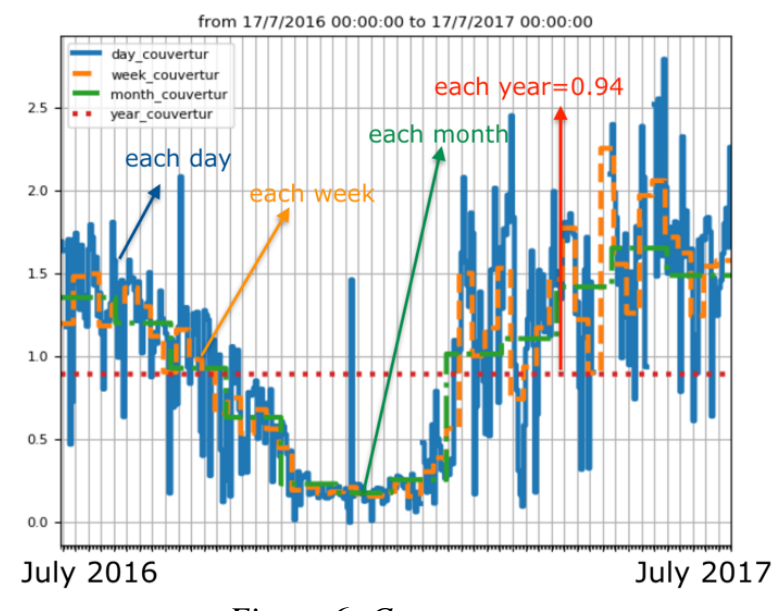

Figure 6: Coverage rate 


\section{From measurement to comparison of predicted simulated values versus measured building performance}

Data from COMEPOS project lead to improve the reliability of the building performance simulation by identifying the potential deviation sources between predicted and measured building performances. Nevertheless, the model validation by the comparison between measured building behavior and the simulation results is not straightforward.

First, the comparison has to be made with the same boundary conditions and context. In that way, meteorological data and occupant behavior in the real and simulated building have to be close. In that way the section "Some global data analyses resulting from measurements" illustrates capabilities of the data processing to extract key information on occupant behavior. Especially for the understanding of the variability of occupant behavior and their impact on building performance. See Gondian et al.(2019) for an original methodology applied on one of the COMEPOS single house.

Generally, the simulation involves several physical phenomena and consequently a large number of parameters and assumptions. They are always associated with some uncertainty or a lack of knowledge. Some inputs, and their uncertainty, have significant impact on the building performance estimation and have to be estimated with care.

To prioritize the characterization effort on the hundreds of building parameters, a sensitivity analysis might be applied. This stochastic method identifies inputs responsible for the lack of robustness in building simulation. For example, Morris screening aims to rank uncertain inputs by influence on the studied output. Global sensitivity analysis associated with uncertainty analysis evaluate first the robustness of the simulation results with a confidence interval and then quantify the influence of each uncertain input on the result. RBDFAST (Goffart et al, 2015), for example, performs the study with only one dataset of hundreds simulations.

Such methods are essential to simplify model complexity and may help to calibrate model by reducing the number of parameter identification. Indeed, the calibration of a whole building performance simulation model is a challenge because of the high number of parameter and the interaction between the building and the solicitations as weather data and occupant behavior. The use of sensitivity analysis and measured data may lead to identify suitable measurement periods for the estimation of each significant factor. Indeed, depending on the configuration (solicitations and states of the building), the influence of the model parameters could drastically change and, thus, affect the quality of the calibration stage. A methodology for calibration based on temporal sensitivity analysis is applied on one of COMEPOS house in Recht et al. (2019).

\section{From measurement to default diagnostic and guaranty of performances}

Guarantee of performance (GP) is a big research challenge that is also addressed by the COMEPOS project. In Josse-Buret et al. (2017) and Josse-Buret (2017), GP has been developed, using a method of Morris, for detecting what could the more important parameters influencing the default. Then an ABC-PMC Bayesian algorithm is used to determine what could be the Bayesian distribution of the parameters explaining the default. Studies have been made on an inhabited house, focusing the analysis on absence periods, since it is during such periods, that we can focus on the physical performances and defaults of the system and the parameters, without being disturbed by inhabitants' behaviour. In Josse-Buret (2017), 2 techniques for absence detection (based typically on the measure of $\mathrm{CO}_{2}$ concentration) were tested and compared. Finally, the impact of simulation on short periods with Morris' screening method has been studied. It finally resulted with a methodology with 4 main steps:

- First, we identify periods where occupants are absent to focus on data that are the less influenced by occupants' behaviour. The analysis of electric data was pointed out as a reliable way to do that.

- Secondly, we reinject inside our model weather parameters and electrical equipment consumptions that are measured in order to minimize the bias due to those parameters.

- Then, we perform an analysis of Morris on the root mean square error (RMSE) of comfort and energy consumption in order to reduce the list of input factors for the calibration process.

- Finally, we apply the Bayesian algorithm ABCPMC to evaluate the range where the identified factors thanks to the Morris's method should be to fit the measurements.

We applied this methodology on cases coming from the COMEPOS demonstrators by using measurements and simulation, we first demonstrated that it is possible to identify absence period thanks to the study of consumption meters. Secondly, we showed at which theoretical conditions, it is possible to detect defaults from global defaults (like a global insulation default), to local default (like a local insulation default of a wall). This result in the development of a software tool called REFATEC and described in Josse-Buret (2017).

\section{Conclusion}

The paper aims to give an overview of the COMEPOS dedicated to "Energy-plus detached houses". This project innovates by proposing optimization and integration of technological solutions to achieve the 'positive energy balance' goal. It succeeds by monitoring and validating the performance of demonstrators under actual use conditions.

From an economic and social point of view, the project will give rise to the development of a model generic economic aiming at the control of the bound additional 
costs with the evolutions of the performances of buildings. The paper focuses more on the scientific and technical developments made for using simulation for design, exploitation of measurement for making sensitivity calculation, calculation of indicators or guaranty of performances.

\section{Acknowledgement}

The project acknowledges the French ADEME Agency for the funding of the project.

\section{References}

Brun, A., Spitz, C., Wurtz, E. and Mora L. (2009). Behavioural comparison of some predictive tools used in a low-energy building. Eleventh International IBPSA Conference. Glasgow (Scotland), 27-30 July 2009.

Deb, K., Pratap, A., Agarwal, S. and Meyarivan, T. (2002). A fast and elitist multiobjective genetic algorithm: NSGA-II. Evolutionary Computation, IEEE Transactions 6(2), 182-197.

Faggianelli, G. A., L. Mora et R. Merheb (2017). « Uncertainty quantification for Energy Savings Performance Contracting : Application to an office building ». Energy and Buildings (152), 61-72.

Goffart, J., Rabouille, M. and Mendes, N. (2015). Uncertainty and sensitivity analysis applied to hygrothermal simulation of a brick building in a hot and humid climate. Journal of Building Performance Simulation 10(1), 37-57.

Gondian, L., Goffart, J., Woloszyn, M., Wurtz, E., Buhé, C. and Maréchal P. (2019). Towards Assessing Houses Resistance and Resilience Indicators At Inhabitants' Actions Using Temporal Sensitivity Analysis. IBPSA 2019. Roma (Italy), 2-4 Sept 2019 [under review].

Josse-Buret, R., Wurtz, F., Maréchal, P. and Wurtz, E. (2017). A Residential Case Study To Validate A New Default Detection Method Based On Discrepancies Between Simulated and Measured Data. IBPSA 2017. San Fancisco (USA), 7-9 August 2017.

Josse-Buret, R. (2017). Méthode et outils pour l'identifi cation de défauts des bâtiments connectés performants. PhD thesis. Univ. Grenoble Alpes,

Morris, M.D. (1991). Factorial sampling plans for preliminary computational experiments. Technometrics 33 (2), 161-174.

Munaretto, F., Peuportier, B. and Guiavarch., A. (2013). Accounting for thermal mass in thermal simulation tools, comparison of several assumptions . Proceedings from BS2013. Chambéry (France).

Munaretto, F., Recht, T., Schalbart, P. and Peuportier, B. (2018). Empirical validation of different internal superficial heat transfer models on a full-scale passive house. Journal of Building Performance Simulation 11 (3), 261-282.
Peuportier, B., and Blanc-Sommereux, I. (1990). Simulation tool with its expert interface for the thermal design of multizone buildings. International Journal of Solar Energy 8(2), 109-120.

Polster, B. (1995). Contribution à l'étude de l'impact environnemental des bâtiments par analyse du cycle de vie. PhD thesis. École nationale supérieure des mines de Paris.

Recht, T., Munaretto, F., Schalbart, P. and Peuportier, B. (2014). Analyse de la fiabilité de COMFIE par comparaison à des mesures. Application à un bâtiment passif. Proceedings from IBPSA France. Arras (France).

Recht, T. (2016). Étude de l'écoconception de maisons à énergie positive. PhD thesis. PSL Research University. https://www.theses.fr/2016PSLEM024

Recht, T., Schalbart, P. and Peuportier, B. (2016). Ecodesign of a "plus-Energy" House Using Stochastic Occupancy Model, Life-Cycle Assessment and Multi-Objective Optimisation. Building Simulation \& Optimisation. Newcastle (UK).

Recht, T., Goffart, J., Mora., L., Woloszyn, M. and Buhé, C. (2019). Predicted And Measured Performances Of Near Zero-Energy Houses: a global comparison methodology. IBPSA 2019. Roma (Italy), 2-4 Sept 2019 [under review].

Salom, J., Marszal, A.J., Widen, J., Candanedo, K. and Byskov Lindberg, K. (2014). Analysis of load match and grid interaction indicators in net zero energy buildings with simulated and monitored data. Applied Energy 136, 119-131.

Vasileios, M., Tsangrassoulis, A. and Axarli, K. (2014). Algorithms for optimization of building design: A review. Renewable and Sustainable Energy Reviews 31, 101-112.

Vorger, E., Schalbart, P. and Peuportier, B. (2014). Integration of a comprehensive stochastic model of occupancy in building simulation to study how inhabitants influence energy performance. Proceedings from PLEA 2014. Ahmedabad (India). 